\title{
Antoine BROCHET
}

\author{
Les résistances territorialisées aux réformes de modernisation des services d'eau. Le \\ cas de l'agglomération grenobloise.
}

\author{
Thèse en Aménagement-Urbanisme de I'Université Grenoble-Alpes, laboratoire PACTE, \\ soutenue le 10 novembre 2017 \\ Directeur de thèse : Bernard Pecqueur
}

\begin{abstract}
Problématique
Résumé

Depuis une vingtaine d'années, les politiques de l'eau potable ont fortement évolué. Suivant un mouvement d'européanisation, ces politiques ont été redéfinies autour de trois objectifs dits « de modernisation » : la libéralisation du secteur ${ }^{1}$, la rationalisation de l'organisation ${ }^{2}$, le développement durable des services d'eau ${ }^{3}$. Les enjeux associés aux réformes de modernisation sont désormais bien connus et étudiés par les chercheurs en sciences sociales, notamment les économistes et gestionnaires. Par contre, la question de la capacité des réformes à produire les effets escomptés demeure un angle mort des recherches.
\end{abstract}

La thèse d'Antoine Brochet est une contribution en ce sens. La problématique étudiée est celle de la territorialisation des réformes de modernisation. Plus précisément, Antoine Brochet propose d'analyser les résistances observées dans la mise en œuvre des réformes de modernisation des services d'eau. Dit autrement, il s'agit de caractériser les désalignements entre d'un côté la philosophie gestionnaire des réformes, et d'un autre côté le substrat technique et les modèles cognitifs des acteurs territoriaux.

\section{Points forts et résultats}

\section{1) La caractérisation et l'analyse des réformes de modernisation des services d'eau}

Un premier point fort de la thèse d'Antoine Brochet est de rendre visible la structure des réformes et leur articulation en trois dimensions (légale, politique et organisationnelle) et deux objectifs principaux : le renforcement de l'efficience du secteur de l'eau (libéralisation du secteur + rationalisation de l'organisation) et l'atteinte d'objectifs environnementaux par les services. Une autre force du travail de recherche concerne la visée opérationnelle de la thèse. Antoine Brochet propose une méthode dénommée ABAFAM, qui permet de simuler l'application des réformes de modernisation à l'échelle d'un ou d'un ensemble de services d'eau potable.

\section{2) La caractérisation des résistances territorialisées aux réformes de modernisation}

Un des principaux résultats de la thèse est la proposition d'un cadre d'analyse pour l'évaluation des résistances territorialisées à la mise en œuvre des réformes de modernisation. Antoine Brochet propose une méthode quantitative permettant de quantifier les résistances mais aussi un répertoire des facteurs de résistance aux réformes de modernisation qui permet de les qualifier et d'en comprendre la nature. Le test de ce répertoire à l'échelle de l'agglomération grenobloise a permis de clarifier l'origine des résistances en montrant que certaines s'expliquent par l'architecture institutionnelle incomplète adossée aux réformes et que d'autres sont directement inscrites dans les modalités de gestion territoriale de l'eau.

3) La compréhension des trajectoires historiques d'accès à l'eau potable dans l'agglomération grenobloise

\footnotetext{
${ }^{1}$ Les politiques de libéralisation se traduisent pour les services d'eau potable par : la désintégration du secteur, le recours aux outils contractuels ou encore l'immixtion du droit privé au sein du droit des services publics.

2 La rationalisation de l'organisation se traduit pour les services d'eau potable par : le retrait des services déconcentrés de l'Etat, la mise en place d'un gouvernement à distance des services d'eau, le changement d'échelle de gestion des services d'eau, etc.

3 L'objectif de durabilité se traduit pour les services d'eau potable par : la mise en place d'une gestion préventive et à la source des problèmes environnementaux, par la mise en place du principe de full cost pricing, ou encore par des dispositifs d'accès social à l'eau.
} 
Trois points forts se dégagent à ce niveau. Le premier point fort est la proposition d'un cadre d'analyse historique multi-niveaux pour évaluer la mise en œuvre des politiques de l'eau à l'échelle des services d'eau potable de l'agglomération grenobloise. Les résultats de la recherche ont permis de nuancer la vision dominante des travaux académiques suivant laquelle l'État a été le principal moteur de développement des services d'eau. L'Etat apparaît dans l'histoire de l'eau de l'agglomération grenobloise uniquement comme un acteur puissant parmi d'autres et en aucun cas comme l'initiateur des décisions hydriques. Un deuxième point fort de l'étude historique est la prise en compte de la dimension de pouvoir dans l'analyse. Antoine Brochet montre que l'histoire de la gestion de l'eau dans l'agglomération grenobloise ne se réduit ni à celle des innovations techniques ni à celle d'une rationalisation progressive de la gestion. À chaque redistribution du pouvoir hydrique dans l'agglomération, la représentation des problèmes, les solutions privilégiées ainsi que les normes de gestion de l'eau ont évolué. Le troisième point fort de l'étude historique est lié à la dimension microcomparative de l'analyse qui a permis de restituer dans sa complexité, l'histoire des services d'eau du bassin grenoblois. Antoine Brochet a ainsi mis en évidence l'existence d'une « hydro diversité territoriale " dans l'agglomération grenobloise, c'est-à-dire l'existence de multiples modèles de gestion de l'eau très territorialisés 4 .

\section{4) La compréhension du phénomène de changement d'échelle des services d'eau}

Une autre force du travail d'Antoine Brochet est la contestation d'un des postulats des réformes de modernisation suivant lequel le changement d'échelle des services d'eau serait vertueux par nature et produirait des effets automatiques et mécaniques : économies d'échelles, meilleure efficacité et efficience du secteur, rationalisation de la gestion de l'eau, etc. Au contraire, l'analyse proposée par le chercheur a permis de nuancer la capacité du pouvoir intercommunal à fédérer les communes membres et à rationaliser la gestion de l'eau du fait des autonomies locales préexistantes. Un autre résultat important de la recherche est la comparaison détaillée des éléments ayant conduit à l'adoption d'une Société Publique Locale (SPL) pour la gestion de l'eau dans les Métropoles de Grenoble, Rennes et Brest. II s'agit d'une contribution utile pour comprendre les ressorts du consentement à cette forme de corporisation qui est nouvelle en France. Enfin, Antoine Brochet propose une réflexion stimulante sur les liens étroits existants entre résistance territoriale et innovation sociale. La thèse montre comment le processus de résistance territoriale analysé dans l'agglomération grenobloise a permis la mise en place d'une organisation innovante et durable de gestion de l'eau permettant de répondre à des enjeux multiples (politiques, économiques, techniques, etc.) liés à la transition environnementale.

\section{Lien de téléchargement de la thèse : http://www.theses.fr/2017GREAH023}

\footnotetext{
4 Depuis près de deux siècles, des petits services d'eau montagnards fonctionnant sous un régime proche de la gestion en bien commun coexistent aux côtés de grands services urbains porteurs de valeurs publiques. Ceci entraîne une fragmentation très forte ainsi qu'un pluralisme des normes de gestion de l'eau à l'échelle de l'agglomération.
} 